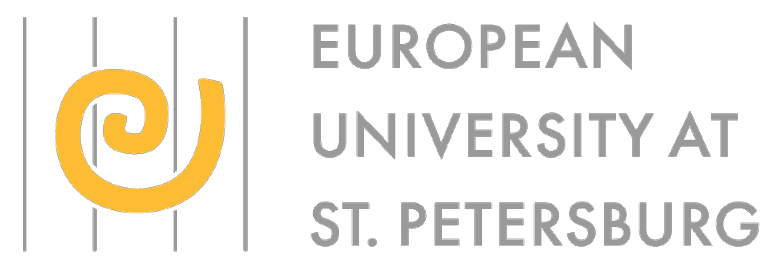

\title{
Local Bifurcations of Three and Four-Dimensional Systems: \\ A Tractable Characterization with Economic Applications
}

by Stefano Bosi and David Desmarchelier

$2017 / 02$

Working Paper Series

Department of Economics

European University at St. Petersburg 
Bosi S., Desmarchelier D. (2017) Local Bifurcations of Three and FourDimensional Systems: A Tractable Characterization with Economic Applications. European University at St. Petersburg, Department of Economics, Working Paper 2017/02, 25 p.

Abstract: We provide necessary and sufficient conditions to detect local bifurcations of three and four-dimensional dynamical systems in continuous time. We characterize the bifurcations of codimension one and two. Our methodology is both general and tractable. To illustrate its operability, we provide two analytical applications of dimension three and four to environmental economics, complemented with numerical simulations.

Keywords: Local bifurcations, Codimensions one and two, Pollution, Natural capital

JEL classification: C61, E32, O44

Stefano Bosi,

EPEE, University of Evry.

David Desmarchelier,

BETA, University of Lorraine.

(C) S. Bosi, D. Desmarchelier, 2017 


\title{
Local bifurcations of three and four-dimensional systems: a tractable characterization with economic applications*
}

\author{
Stefano BOSI \\ EPEE, University of Evry
}

\author{
David DESMARCHELIER \\ BETA, University of Lorraine
}

16th June 2017

\begin{abstract}
We provide necessary and sufficient conditions to detect local bifurcations of three and four-dimensional dynamical systems in continuous time. We characterize the bifurcations of codimension one and two. Our methodology is both general and tractable. To illustrate its operability, we provide two analytical applications of dimension three and four to environmental economics, complemented with numerical simulations.

Keywords: local bifurcations, codimensions one and two, pollution, natural capital.
\end{abstract}

JEL Classification: C61, E32, O44.

\section{Introduction}

In dynamic general equilibrium theory, the most popular model is Ramsey (1928). The core of this continuous-time model is a two-dimensional dynamical system. The Ramsey model is characterized by the saddle-path stability of a unique equilibrium. Many dynamic economic models are extensions of the Ramsey model. The introduction of market imperfections or agents' heterogeneity often increases the dimension of the dynamical system and makes dynamics richer: the non-linearities associated to these imperfections change the stability properties of the steady state and generate more complex attractors such as the limit cycles. For example, monetary extensions of a Ramsey model are

\footnotetext{
* Previous versions of the paper circulate as working papers of the research centers BETA and EPEE (Bosi and Desmarchelier, 2017). The authors would like to thank the participants to the conference "Economic Growth, Macroeconomic Dynamics and Agents' Heterogeneity" held in St. Petersburg on May 2017, and to the Asian Meeting of the Econometric Society held in Hong Kong on June 2017. Stefano Bosi acknowledges the financial support of the LABEX MME-DII.
} 
three-dimensional while two-country general equilibrium models are often fourdimensional. In general, the introduction of an additional building block in some previous extension of the Ramsey model raises the dimension of the dynamical system and makes the economic analysis more difficult.

The stability change of a dynamical system corresponds to a change in some fundamental parameter through a critical value. When the parameter crosses a critical value, a bifurcation takes place. A bifurcation is said to be local when it arises in a neighborhood of an attractor (such as a steady state). If the bifurcation is generated by one parameter only, it is said of codimension one. When the bifurcation is generated by the joint change of two parameters, it is said of codimension two.

Our paper addresses the methodological question of necessary and sufficient conditions for local bifurcations of higher-dimensional dynamical systems in continuous time. We provide a simple method to detect bifurcations of codimension one and two in the case of three and four-dimensional dynamical systems using the sum of minors of the Jacobian matrix.

We do not consider two-dimensional systems because the characterization of local bifurcations is known. Kuznetsov and Sedova (2012) treats also the case of local bifurcations of three and four-dimensional maps in discrete time. Barinci and Drugeon (2017) apply a geometrical method to characterize the local bifurcations of three-dimensional maps in discrete time. In continuous time, Dockner and Feichtinger (1991) provide conditions for a Hopf bifurcation to occur in the specific case of a four-dimensional system resulting from a dynamic optimization with two state variables. Our methodology is very easy to handle and applies to general continuous-time systems: unlike Dockner and Feichtinger (1991), it applies to any kind of three or four-dimensional systems of autonomous ODE. To illustrate our approach we give two basic examples of environmental economies of dimension three and four respectively. To convince the reader, the analytical characterizations are complemented by numerical simulations based on the original non-linear systems.

The rest of the paper is organized as follows. Section 2 characterizes the local bifurcations of three-dimensional dynamical system and provides an economic example with pollution, while Section 3 focuses instead on four-dimensional systems and gives an example with natural capital. Both the economic illustrations of our methodology are complemented with numerical simulations. All the proof are gathered in the Appendix.

\section{Local bifurcation of three-dimensional system}

We consider dynamics driven by a system of three autonomous Ordinary Differential Equations (ODE):

$$
\begin{aligned}
& \dot{x}_{1}=f_{1}\left(x_{1}, x_{2}, x_{3}\right) \\
& \dot{x}_{2}=f_{2}\left(x_{1}, x_{2}, x_{3}\right) \\
& \dot{x}_{3}=f_{3}\left(x_{1}, x_{2}, x_{3}\right)
\end{aligned}
$$


We linearize the system around a steady state $\left(x_{1}, x_{2}, x_{3}\right)^{*}$. The Jacobian matrix is given by

$$
J \equiv\left[\begin{array}{lll}
\frac{\partial f_{1}}{\partial x_{1}} & \frac{\partial f_{1}}{\partial x_{2}} & \frac{\partial f_{1}}{\partial x_{3}} \\
\frac{\partial f_{2}}{\partial x_{1}} & \frac{\partial f_{2}}{\partial x_{2}} & \frac{\partial f_{2}}{\partial x_{3}} \\
\frac{\partial f_{3}}{\partial x_{1}} & \frac{\partial f_{3}}{\partial x_{2}} & \frac{\partial f_{3}}{\partial x_{3}}
\end{array}\right]
$$

We define the sums of principal minors of order one, two and three:

$$
\begin{aligned}
S_{1} & \equiv \frac{\partial f_{1}}{\partial x_{1}}+\frac{\partial f_{2}}{\partial x_{2}}+\frac{\partial f_{3}}{\partial x_{3}} \\
S_{2} & \equiv\left|\left[\begin{array}{ll}
\frac{\partial f_{2}}{\partial x_{2}} & \frac{\partial f_{2}}{\partial x_{3}} \\
\frac{\partial f_{3}}{\partial x_{2}} & \frac{\partial f_{3}}{\partial x_{3}}
\end{array}\right]\right|+\left|\left[\begin{array}{ll}
\frac{\partial f_{1}}{\partial x_{1}} & \frac{\partial f_{1}}{\partial x_{3}} \\
\frac{\partial f_{3}}{\partial x_{1}} & \frac{\partial f_{3}}{\partial x_{3}}
\end{array}\right]\right|+\left|\left[\begin{array}{cc}
\frac{\partial f_{1}}{\partial x_{1}} & \frac{\partial f_{1}}{\partial x_{2}} \\
\frac{\partial f_{2}}{\partial x_{1}} & \frac{\partial f_{2}}{\partial x_{2}}
\end{array}\right]\right| \\
S_{3} & \equiv\left|\left[\begin{array}{lll}
\frac{\partial f_{1}}{\partial x_{1}} & \frac{\partial f_{1}}{\partial x_{2}} & \frac{\partial f_{1}}{\partial x_{3}} \\
\frac{\partial f_{2}}{\partial x_{1}} & \frac{\partial f_{2}}{\partial x_{2}} & \frac{\partial f_{2}}{\partial x_{3}} \\
\frac{\partial f_{3}}{\partial x_{1}} & \frac{\partial f_{3}}{\partial x_{2}} & \frac{\partial f_{3}}{\partial x_{3}}
\end{array}\right]\right|
\end{aligned}
$$

We denote $S_{1}$ by $T$ (trace), $S_{2}$ by $S$, and $S_{3}$ by $D$ (determinant). Clearly, the values taken by $T, S$ and $D$ depend on the steady state $x^{*}$ we focus on.

We know that, in terms of information, the vector $(T, S, D)$ is equivalent to the vector of eigenvalues $\left(\lambda_{1}, \lambda_{2}, \lambda_{3}\right)$. More precisely, we have $T=\lambda_{1}+\lambda_{2}+\lambda_{3}$, $S=\lambda_{1} \lambda_{2}+\lambda_{1} \lambda_{3}+\lambda_{2} \lambda_{3}$ and $D=\lambda_{1} \lambda_{2} \lambda_{3}$. The characteristic polynomial of our three-dimensional dynamics becomes

$$
P(\lambda)=\left(\lambda-\lambda_{1}\right)\left(\lambda-\lambda_{2}\right)\left(\lambda-\lambda_{3}\right)=\lambda^{3}-T \lambda^{2}+S \lambda-D
$$

\subsection{Codimension one}

In continuous time, a local bifurcation generically arises when the real part of an eigenvalue $\lambda(p)$ of the Jacobian matrix crosses zero in response to a change in a parameter $p$. Denoting by $p^{*}$ the critical parameter value of bifurcation, we get generically two cases: (1) when a real eigenvalue crosses zero: $\lambda\left(p^{*}\right)=0$, the system undergoes a saddle-node bifurcation (either an elementary saddlenode or a transcritical or a pitchfork bifurcation depending on the number of steady states), (2) when the real part of two complex and conjugate eigenvalues $\lambda(p)=a(p) \pm i b(p)$ crosses zero, the system undergoes a Hopf bifurcation. More precisely, in the second case, we require $a\left(p^{*}\right)=0$ and $b(p) \neq 0$ in a neighborhood of $p^{*}$ (see, for local bifurcations in continuous time, Bosi and Ragot, 2011, p. 76, and, in discrete time, Grandmont, 2008).

\subsubsection{Saddle node bifurcation}

When a real eigenvalue crosses zero, a bifurcation of the saddle-node family takes place. In the case of an elementary saddle node, two steady states (one stable, the other unstable) coalesce and disappear. In the case of a transcritical bifurcation, two steady states (one stable, the other unstable) coalesce and then 
separate again while exchanging their stability properties. Finally, in the case of a pitchfork bifurcation, three steady states coalesce into one: a stable (unstable) steady state surrounded by two unstable (stable) becomes unstable (stable).

Proposition 1 (saddle-node) A saddle-node arises if and only if $D=0$.

\subsubsection{Hopf bifurcation}

A Hopf bifurcation generates limit cycles either attractive (supercritical) or repulsive (subcritical).

Proposition 2 (Hopf) In the case of a three-dimensional system, a Hopf bifurcation generically arises if and only if $D=S T$ and $S>0$.

\subsection{Codimension two}

For now, we have considered only one bifurcation parameter. The codimension of a bifurcation is the number of parameters to vary for the bifurcation to occur (see Kuznetsov (1998) among others).

Focus on a pair of scalar parameters: $p$ and $q$. Assume that, given $p$, two bifurcations occur at $q=q_{1}(p)$ and $q=q_{2}(p)$. If the bifurcation curves $q_{1}$ and $q_{2}$ obtained varying $p$ cross at $(p, q)^{*}$ in the $(p, q)$-plane, where $q^{*}=q_{1}\left(p^{*}\right)=$ $q_{2}\left(p^{*}\right)$, then the dynamical system generically experiences a codimension-two bifurcation (see Kuznetsov (1998) among others).

\subsubsection{Bogdanov-Takens bifurcation}

Assume that the system (1) possesses two steady states: the first one is a saddle point while the second is surrounded by a limit cycle. A BogdanovTakens bifurcation arises when the cycle coalesces with the saddle point. When this bifurcation occurs, the limit cycle disappears and a so-called parasitic loop arises (Kuznetsov, 1998).

Definition 3 (Bogdanov-Takens) Consider the curve in a parametric $(p, q)$ plane along which a real eigenvalue $\lambda_{1}$ remains equal to zero. Assume that, when the pair $(p, q)$ moves along this curve, an additional real eigenvalues $\lambda_{2}$ becomes zero at $(p, q)^{*}$. In this case, the central manifold becomes two-dimensional and a Bogdanov-Takens (or double-zero) bifurcation arises at $(p, q)^{*}$.

Proposition 4 (Bogdanov-Takens) A Bogdanov-Takens bifurcation generically occurs if and only if $D=S=0$.

\subsubsection{Gavrilov-Guckenheimer bifurcation}

A Gavrilov-Guckenheimer (also called zero-Hopf) bifurcation corresponds to the intersection of two bifurcations curves: Hopf and saddle-node. Differently from the Bogdanov-Takens bifurcation, the limit cycle is preserved and its interaction with the saddle-node bifurcation can lead to richer dynamics including the cases of invariant torus and local chaos (Kuznetsov, 1998). 
Definition 5 (Gavrilov-Guckenheimer) A Gavrilov-Guckenheimer bifurcation arises when $\lambda_{1}=0, \lambda_{2}=b i=-\lambda_{3}$.

Proposition 6 (Gavrilov-Guckenheimer) A Gavrilov-Guckenheimer bifurcation occurs if and only if $D=T=0$ jointly with $S>0$.

\subsection{Economic example with pollution}

We consider a simple Ramsey economy where a pollution externality, coming from the use of capital at the firm level, affects the marginal utility of consumption. This economy is very close to Heal (1982) and Bosi and Desmarchelier (2016a).

A firm $j$ uses a Cobb-Douglas production function to produce a single commodity $Y_{j}(t)=A K_{j}(t)^{\alpha} L_{j}(t)^{1-\alpha}$ where $K_{j}$ and $L_{j}$ are respectively the amount of capital and labor. $K \equiv \sum_{j} K_{j}$ and $L \equiv \sum_{j} L_{j}$ denote the aggregate inputs. $A>0$ captures the total factor productivity while $\alpha \in(0,1)$ represents the capital share in total income. For notational parsimony, the time argument $t$ will be omitted in the following. Firm chooses the amount of capital and labor to maximize the profit taking as given the real interest rate $r$ and the real wage $w$. All the firms share the same technology.

A representative household earns a capital income $r h$ and a labor income $w l$ where $h$ and $l$ denote the individual wealth and labor supply at time $t$. For simplicity, we assume also that the household supplies inelastically one unit of labor: $l=1$. Thus, the household consumes and saves its income according to the budget constraint

$$
c+\dot{h} \leq(r-\delta) h+w
$$

where $\dot{h}$ denotes the time-derivative of wealth while $c$ represents the consumption level. The gross investment includes the capital depreciation at the rate $\delta$. For the sake of simplicity, the population of consumers-workers is constant over time and normalized to one: $n=1$. Such a normalization implies $L=n l=l=1$, $K=n h=h$ and $h=K / n=k l=k$, where $k \equiv K / L$ denotes the capital intensity.

In a Ramsey model, the representative household maximizes an intertemporal utility functional

$$
\int_{0}^{\infty} e^{-\rho t} \frac{\left(c P^{-\eta}\right)^{1-\varepsilon}}{1-\varepsilon} d t
$$

under the budget constraint (3) where $\rho>0$ denotes the rate of time preference. $P$ represents the pollution level, a pure externality, while $1 / \varepsilon$ is the usual consumption elasticity of intertemporal substitution and $\eta>0$ is a measure of the household's environmental concern.

The aggregate stock of pollution $P$ is a pure externality coming from the aggregate capital stock used to produce $(K)$. To take things as simple as possible, we assume a linear process:

$$
\dot{P}=-a P+b K
$$


$a>0$ and $b>0$ capture the natural rate of pollution absorption and the environmental impact of capital.

Proposition 7 (general equilibrium) Equilibrium dynamics are represented by the following system:

$$
\begin{aligned}
\dot{\mu} & =f_{1}(\mu, k, P) \equiv \mu\left(\rho+\delta-\alpha A k^{\alpha-1}\right) \\
\dot{k} & =f_{2}(\mu, k, P) \equiv\left(\alpha A k^{\alpha-1}-\delta\right) k+(1-\alpha) A k^{\alpha}-\mu^{-\frac{1}{\varepsilon}} P^{\eta \frac{\varepsilon-1}{\varepsilon}} \\
\dot{P} & =f_{3}(\mu, k, P) \equiv-a P+b k
\end{aligned}
$$

where $\mu$ is the shadow price of capital (marginal utility of consumption).

Proposition 8 (steady state) A steady state exists. The steady state is unique:

$$
k^{*}=\left(\frac{\alpha A}{\rho+\delta}\right)^{\frac{1}{1-\alpha}}, P^{*}=\frac{b}{a} k^{*} \text { and } \mu^{*}=\left[\rho k^{*}+(1-\alpha) A k^{* \alpha}\right]^{-\varepsilon} P^{* \eta(\varepsilon-1)}
$$

To study the local bifurcations, we linearize the system (4)-(6) around this unique steady state.

Lemma 9 The sums of minors of order one (trace), two and three (determinant) of the Jacobian matrix are respectively given by

$$
\begin{aligned}
T & =\rho-a \\
S & =\varphi[a \eta(\varepsilon-1)-(1-\alpha)(\rho+\delta)]-a \rho \\
D & =a \varphi(1-\alpha)(\rho+\delta)
\end{aligned}
$$

where

$$
\varphi \equiv \frac{\rho+(1-\alpha) \delta}{\alpha \varepsilon}
$$

Proposition 10 (saddle-node, BT and GG) In this example, saddle-node, Bogdanov-Takens and Gavrilov-Guckenheimer bifurcations are impossible.

Remark 11 In order to convince the reader about the impossibility of saddle node bifurcations, we observe that all the bifurcations of this family (elementary saddle node, transcritical and pitchfork) always involve multiple steady states (two or three). In our example, the steady state is unique.

Focus now on the occurrence of Hopf bifurcations and the main economic parameter: the measure of households' sensitivity to environmental issues $\eta$.

Proposition 12 (Hopf) Let $a<\rho$ jointly with $\varepsilon>1$. When $\eta$ crosses

$$
\eta_{H} \equiv \frac{\rho}{\varepsilon-1}\left(\frac{1}{\varphi}+\frac{1-\alpha}{a} \frac{\rho+\delta}{\rho-a}\right)
$$

then a limit cycle arises around $(\mu, k, P)^{*}$ through a Hopf bifurcation. 
To study the stability of the limit cycle, we perform a numerical simulation under the following calibration:

\begin{tabular}{||l||l||l||l||l||l||l||l||}
\hline \hline Parameter & $A$ & $\alpha$ & $\rho$ & $\delta$ & $a$ & $b$ & $\varepsilon$ \\
\hline \hline Value & 1 & 0.3 & 0.2 & 0.1 & 0.1 & 0.1 & 2 \\
\hline \hline
\end{tabular}

To simplify the computations, we have fixed $A, \alpha, \rho$ and $\delta$ such that $k^{*}=1$. In addition, we have set the discounting sufficiently high $(\rho>a)$ to ensure a positive trace. A more empirically plausible calibration is given in Bosi and Desmarchelier (2016a), that is a more realistic model with environmental maintenance and green taxation. Our values allows also MATCONT to compute easily the orbit at the Hopf bifurcation point using the original non-linear system. ${ }^{1}$ Finally, we observe that $\varepsilon>1$ and $\rho>a$ are necessary (but not sufficient) conditions for the occurrence of a Hopf bifurcation (according to Proposition 12).

According to Proposition 12, a Hopf bifurcation arises at $\eta=\eta_{H}=4.6444$. At the Hopf bifurcation point, the steady state becomes $(\mu, k, P)^{*}=(1.2346,1,1)$ and the eigenvalues are given by $\lambda_{1}=0.307409 i=-\lambda_{2}$ and $\lambda_{3}=0.1$.

The corresponding first Lyapunov coefficient is negative $l_{1}=-0.1249661<$ 0 . This implies that the Hopf bifurcation is supercritical, that is the limit cycle arising near the steady state is stable (Fig. 1).

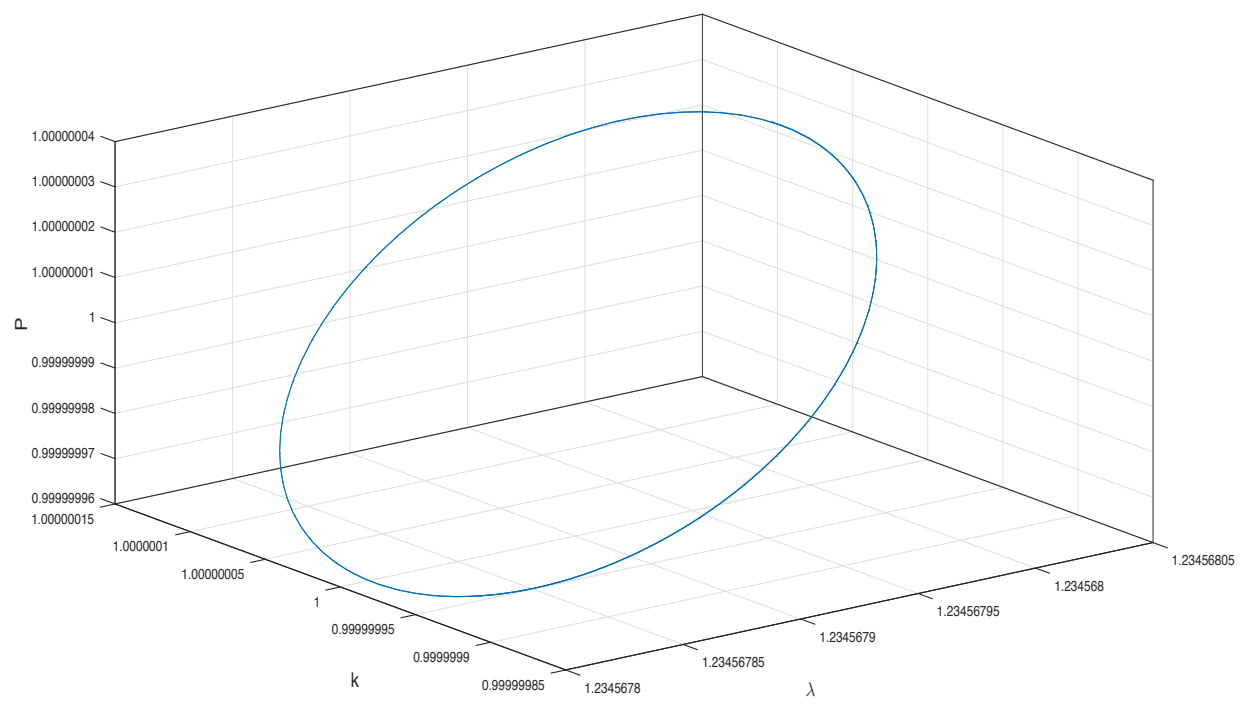

Fig. 1. Stable limit cycle.

Focus now on a higher-dimensional example.

${ }^{1}$ We use the MATCONT package for MATLAB version $6 \mathrm{p} 6$. 


\section{Local bifurcations of four-dimensional system}

We consider now dynamics driven by a system of four autonomous Ordinary Differential Equations (ODE):

$$
\begin{aligned}
& \dot{x}_{1}=f_{1}\left(x_{1}, x_{2}, x_{3}, x_{4}\right) \\
& \dot{x}_{2}=f_{2}\left(x_{1}, x_{2}, x_{3}, x_{4}\right) \\
& \dot{x}_{3}=f_{3}\left(x_{1}, x_{2}, x_{3}, x_{4}\right) \\
& \dot{x}_{4}=f_{4}\left(x_{1}, x_{2}, x_{3}, x_{4}\right)
\end{aligned}
$$

We linearize the system around a steady state $\left(x_{1}, x_{2}, x_{3}, x_{4}\right)^{*}$. The Jacobian matrix is given by

$$
J \equiv\left[\begin{array}{llll}
\frac{\partial f_{1}}{\partial x_{1}} & \frac{\partial f_{1}}{\partial x_{2}} & \frac{\partial f_{1}}{\partial x_{3}} & \frac{\partial f_{1}}{\partial x_{4}} \\
\frac{\partial f_{2}}{\partial x_{1}} & \frac{\partial f_{2}}{\partial x_{2}} & \frac{\partial f_{2}}{\partial x_{3}} & \frac{\partial f_{2}}{\partial x_{4}} \\
\frac{\partial f_{3}}{\partial x_{1}} & \frac{\partial f_{3}}{\partial x_{2}} & \frac{\partial f_{3}}{\partial x_{3}} & \frac{\partial f_{3}}{\partial x_{4}} \\
\frac{\partial f_{4}}{\partial x_{1}} & \frac{\partial f_{4}}{\partial x_{2}} & \frac{\partial f_{4}}{\partial x_{3}} & \frac{\partial f_{4}}{\partial x_{4}}
\end{array}\right]
$$

We define the sums of principal minors of order one, two, three and four:

$$
\begin{aligned}
& S_{1} \equiv \frac{\partial f_{1}}{\partial x_{1}}+\frac{\partial f_{2}}{\partial x_{2}}+\frac{\partial f_{3}}{\partial x_{3}}+\frac{\partial f_{4}}{\partial x_{4}} \\
& S_{2} \equiv\left|\left[\begin{array}{ll}
\frac{\partial f_{3}}{\partial x_{3}} & \frac{\partial f_{3}}{\partial x_{4}} \\
\frac{\partial f_{4}}{\partial x_{3}} & \frac{\partial f_{4}}{\partial x_{4}}
\end{array}\right]\right|+\left|\left[\begin{array}{ll}
\frac{\partial f_{2}}{\partial x_{2}} & \frac{\partial f_{2}}{\partial x_{4}} \\
\frac{\partial f_{4}}{\partial x_{2}} & \frac{\partial f_{4}}{\partial x_{4}}
\end{array}\right]\right|+\left|\left[\begin{array}{ll}
\frac{\partial f_{2}}{\partial x_{2}} & \frac{\partial f_{2}}{\partial x_{3}} \\
\frac{\partial f_{3}}{\partial x_{2}} & \frac{\partial f_{3}}{\partial x_{3}}
\end{array}\right]\right| \\
& +\left|\left[\begin{array}{ll}
\frac{\partial f_{1}}{\partial x_{1}} & \frac{\partial f_{1}}{\partial x_{4}} \\
\frac{\partial f_{4}}{\partial x_{1}} & \frac{\partial f_{4}}{\partial x_{4}}
\end{array}\right]\right|+\left|\left[\begin{array}{ll}
\frac{\partial f_{1}}{\partial x_{1}} & \frac{\partial f_{1}}{\partial x_{3}} \\
\frac{\partial f_{3}}{\partial x_{1}} & \frac{\partial f_{3}}{\partial x_{3}}
\end{array}\right]\right|+\left|\left[\begin{array}{ll}
\frac{\partial f_{1}}{\partial x_{1}} & \frac{\partial f_{1}}{\partial x_{2}} \\
\frac{\partial f_{2}}{\partial x_{1}} & \frac{\partial f_{2}}{\partial x_{2}}
\end{array}\right]\right| \\
& S_{3} \equiv\left|\left[\begin{array}{lll}
\frac{\partial f_{2}}{\partial x_{2}} & \frac{\partial f_{2}}{\partial x_{3}} & \frac{\partial f_{2}}{\partial x_{4}} \\
\frac{\partial f_{3}}{\partial x_{2}} & \frac{\partial f_{3}}{\partial x_{3}} & \frac{\partial f_{3}}{\partial x_{4}} \\
\frac{\partial f_{4}}{\partial x_{2}} & \frac{\partial f_{4}}{\partial x_{3}} & \frac{\partial f_{4}}{\partial x_{4}}
\end{array}\right]\right|+\left|\left[\begin{array}{lll}
\frac{\partial f_{1}}{\partial x_{1}} & \frac{\partial f_{1}}{\partial x_{3}} & \frac{\partial f_{1}}{\partial x_{4}} \\
\frac{\partial f_{3}}{\partial x_{1}} & \frac{\partial f_{3}}{\partial x_{3}} & \frac{\partial f_{3}}{\partial x_{4}} \\
\frac{\partial f_{4}}{\partial x_{1}} & \frac{\partial f_{4}}{\partial x_{3}} & \frac{\partial f_{4}}{\partial x_{4}}
\end{array}\right]\right| \\
& +\left|\left[\begin{array}{lll}
\frac{\partial f_{1}}{\partial x_{1}} & \frac{\partial f_{1}}{\partial x_{2}} & \frac{\partial f_{1}}{\partial x_{4}} \\
\frac{\partial f_{2}}{\partial x_{1}} & \frac{\partial f_{2}}{\partial x_{2}} & \frac{\partial f_{2}}{\partial x_{4}} \\
\frac{\partial f_{4}}{\partial x_{1}} & \frac{\partial f_{4}}{\partial x_{2}} & \frac{\partial f_{4}}{\partial x_{4}}
\end{array}\right]\right|+\left|\left[\begin{array}{lll}
\frac{\partial f_{1}}{\partial x_{1}} & \frac{\partial f_{1}}{\partial x_{2}} & \frac{\partial f_{1}}{\partial x_{3}} \\
\frac{\partial f_{2}}{\partial x_{1}} & \frac{\partial f_{2}}{\partial x_{2}} & \frac{\partial f_{2}}{\partial x_{3}} \\
\frac{\partial f_{3}}{\partial x_{1}} & \frac{\partial f_{3}}{\partial x_{2}} & \frac{\partial f_{3}}{\partial x_{3}}
\end{array}\right]\right| \\
& S_{4} \equiv\left|\left[\begin{array}{llll}
\frac{\partial f_{1}}{\partial x_{1}} & \frac{\partial f_{1}}{\partial x_{2}} & \frac{\partial f_{1}}{\partial x_{3}} & \frac{\partial f_{1}}{\partial x_{4}} \\
\frac{\partial f_{2}}{\partial x_{1}} & \frac{\partial f_{2}}{\partial x_{2}} & \frac{\partial f_{2}}{\partial x_{3}} & \frac{\partial f_{2}}{\partial x_{4}} \\
\frac{\partial f_{3}}{\partial x_{1}} & \frac{\partial f_{3}}{\partial x_{2}} & \frac{\partial f_{3}}{\partial x_{3}} & \frac{\partial f_{3}}{\partial x_{4}} \\
\frac{\partial f_{4}}{\partial x_{1}} & \frac{\partial f_{4}}{\partial x_{2}} & \frac{\partial f_{4}}{\partial x_{3}} & \frac{\partial f_{4}}{\partial x_{4}}
\end{array}\right]\right|
\end{aligned}
$$

We denote $S_{1}$ by $T$ (trace) and $S_{4}$ by $D$ (determinant). Clearly, the values taken by $T, S_{2}, S_{3}$ and $D$ depend on the steady state $x^{*}$ we focus on.

The characteristic polynomial is given by

$$
P(\lambda) \equiv\left(\lambda-\lambda_{1}\right)\left(\lambda-\lambda_{2}\right)\left(\lambda-\lambda_{3}\right)\left(\lambda-\lambda_{4}\right)=\lambda^{4}-T \lambda^{3}+S_{2} \lambda^{2}-S_{3} \lambda+D
$$


where

$$
\begin{aligned}
& S_{1}=\lambda_{1}+\lambda_{2}+\lambda_{3}+\lambda_{4}=T \\
& S_{2}=\lambda_{1} \lambda_{2}+\lambda_{1} \lambda_{3}+\lambda_{1} \lambda_{4}+\lambda_{2} \lambda_{3}+\lambda_{2} \lambda_{4}+\lambda_{3} \lambda_{4} \\
& S_{3}=\lambda_{1} \lambda_{2} \lambda_{3}+\lambda_{1} \lambda_{2} \lambda_{4}+\lambda_{1} \lambda_{3} \lambda_{4}+\lambda_{2} \lambda_{3} \lambda_{4} \\
& S_{4}=\lambda_{1} \lambda_{2} \lambda_{3} \lambda_{4}=D
\end{aligned}
$$

\subsection{Codimension one}

As above, we consider first bifurcations involving only one parameters (codimension one).

\subsubsection{Saddle-node bifurcation}

As seen above, when a real eigenvalue crosses zero, the system undergoes a saddle-node bifurcation (either an elementary saddle-node or a transcritical or a pitchfork bifurcation depending on the number of steady states).

Proposition 13 (saddle-node) A saddle-node bifurcation generically arises when $D=0$.

\subsubsection{Hopf bifurcation}

When the real part of two complex and conjugate eigenvalues with nonzero imaginary part crosses zero, the system undergoes a Hopf bifurcation.

Proposition 14 (Hopf) A Hopf bifurcation generically arises if and only if

$$
S_{2}=\frac{S_{3}}{T}+\frac{D T}{S_{3}}
$$

and $T$ and $S_{3}$ have the same sign.

\subsection{Codimension two}

As seen in the case of three-dimensional systems, when a bifurcation involves two parameters, richer dynamics arise. However, the case of four-dimensional system allows for a new bifurcation involving two coalescing Hopf bifurcations and generating more complex dynamics. Of course, the other bifurcations arising in the three-dimensional case remain possible when the system becomes higherdimensional.

\subsubsection{Bogdanov-Takens bifurcation}

Reconsider Definition 3. A Bogdanov-Takens bifurcation is a double-zero bifurcation: $\lambda_{1}=\lambda_{2}=0$.

Proposition 15 (Bogdanov-Takens) A Bogdanov-Takens bifurcation generically occurs if and only if $S_{3}=D=0$. 


\subsubsection{Gavrilov-Guckenheimer bifurcation}

According to Definition 5, a Gavrilov-Guckenheimer bifurcation arises when $\lambda_{1}=0, \lambda_{2}=b i=-\lambda_{3}$. In the case of a four-dimensional system, our characterization involves not only the trace and the determinant, but also the sums of minors of order two and three.

Proposition 16 (Gavrilov-Guckenheimer) A Gavrilov-Guckenheimer bifurcation generically arises if and only if $D=0$ and $S_{3}=T S_{2}$ with $S_{2}>0$.

\subsubsection{Double-Hopf bifurcation}

The double-Hopf bifurcation occurs at the intersection of two Hopf bifurcation curves in the parameter plane. This bifurcation leads generically to a torus. The double-Hopf bifurcation can imply richer dynamics such as Smale horseshoes and local chaos (see Kuznetsov (1998) among others).

Definition 17 (double-Hopf) $A$ double-Hopf bifurcation occurs when $\lambda_{1}=$ $b i, \lambda_{2}=-b i, \lambda_{3}=d i$ and $\lambda_{4}=-d i$.

Proposition 18 (double-Hopf) A double-Hopf bifurcation generically arises if and only if $T=S_{3}=0$ with $D>0, S_{2}>0$ and $S_{2}^{2} \geq 4 D$.

\subsection{Economic example with natural capital}

We consider a Ramsey economy in the spirit of Wirl (2004) and Bosi and Desmarchelier (2016b). A pollution externality coming from production activities impairs a natural resource which affects the consumption behavior. The very difference with Bosi and Desmarchelier (2016b) is that pollution plays now the role of stock variable instead of flow. This role affects the dimension of dynamical system and, hence, entails important consequences.

For simplicity, firms behave as in the previous example and the same firstorder conditions for profit maximization hold. As above, a representative household chooses a consumption path to maximize its intertemporal utility functional

$$
\int_{0}^{\infty} e^{-\rho t} \frac{\left(c N^{\eta}\right)^{1-\varepsilon}}{1-\varepsilon} d t
$$

with respect to its budget constraint (3), where $N$ denotes now the aggregate natural resource. For the sake of simplicity, the population of consumers-workers is constant over time and normalized to one: $n=1$. Such a normalization implies $L=n l=l=1, K=n h=h$ and $h=K / n=k l=k$, where $k \equiv K / L$ denotes the capital intensity.

The aggregate stock of pollution $P$ is a pure externality coming from production. As above, we consider a linear process, but pollution depends now on aggregate production:

$$
\dot{P}=-a P+b Y
$$


$a>0$ and $b>0$ capture the natural rate of pollution absorption and the environmental impact of production. $K=k$ and $L=1$ imply $\dot{P}=b A k^{\alpha}-a P$.

In the spirit of Ayong Le Kama (2001) and Wirl (2004), the dynamics of natural resource is driven by:

$$
\dot{N}=N(1-N)-\gamma P
$$

where $\gamma>0$ represents the impact of pollution on the natural resource. More precisely, the component $N(1-N)$ is a Pearl-Verhulst logistic function representing the so-called reproduction function of nature. This component attains its maximum at $N=1 / 2$ (maximal sustainable yield).

Proposition 19 (general equilibrium) Equilibrium dynamics are represented by a four-dimensional dynamical system:

$$
\begin{aligned}
\dot{\mu} & =f_{1}(\mu, k, N, P) \equiv \mu\left(\rho+\delta-\alpha A k^{\alpha-1}\right) \\
\dot{k}=f_{2}(\mu, k, N, P) & \equiv A k^{\alpha}-\delta k-\mu^{-\frac{1}{\varepsilon}} N^{\eta \frac{1-\varepsilon}{\varepsilon}} \\
\dot{N} & =f_{3}(\mu, k, N, P) \equiv N(1-N)-\gamma P \\
\dot{P} & =f_{4}(\mu, k, N, P) \equiv b A k^{\alpha}-a P
\end{aligned}
$$

The following proposition addresses both the existence and the multiplicity of steady states.

Proposition 20 (steady states) The steady states are given by

$$
\begin{aligned}
\mu^{*} & =\left(\varepsilon \varphi k^{*}\right)^{-\varepsilon} N_{i}^{(1-\varepsilon) \eta} \\
k^{*} & =\left(\frac{\alpha A}{\rho+\delta}\right)^{\frac{1}{1-\alpha}} \\
P^{*} & =\frac{b A}{a} k^{* \alpha} \\
N_{1} & =\frac{1}{2}\left(1-\sqrt{1-4 \gamma P^{*}}\right) \\
N_{2} & =\frac{1}{2}\left(1+\sqrt{1-4 \gamma P^{*}}\right)
\end{aligned}
$$

where $\varphi$ is given by (7).

Let $b^{*} \equiv a /\left(4 \gamma A k^{* \alpha}\right)$ be the critical environmental impact of production.

If $b<b^{*}$, there are two steady states with $0<N_{1}<1 / 2<N_{2}<1$.

If $b=b^{*}$, the two steady states coalesce: $N_{1}=N_{2}=1 / 2$.

If $b>b^{*}$, there are no steady states.

To study the local bifurcations, we linearize the system (14)-(17) around both the steady states $\left(\mu, k, N_{i}, P\right)^{*}, i=1,2$. 
Lemma 21 The sums of minors of order one (trace), two, three and four (determinant) are respectively given by

$$
\begin{aligned}
T & =\rho-a+1-2 N \\
S_{2} & =(\rho-a)(1-2 N)-a \rho-\varphi(1-\alpha)(\rho+\delta) \\
S_{3} & =z+a \alpha \varphi \eta(\varepsilon-1)(N-1) \equiv S_{3}(\eta) \\
D & =a \varphi(1-\alpha)(\rho+\delta)(1-2 N)
\end{aligned}
$$

with

$$
z \equiv \varphi(1-\alpha)(\rho+\delta)(a+2 N-1)+a \rho(2 N-1)
$$

Notice that only $S_{3}$ depends on $\eta$.

Proposition 22 (elementary saddle node) An elementary saddle-node bifurcation occurs (when $N_{1}$ and $N_{2}$ coalesce) if and only if $b=b^{*}$.

$\eta$ captures the importance of nature for households and, so, the specificity of our model. It makes sense to consider it as main bifurcation parameter. We introduce two critical values to study the occurrence of Hopf bifurcations:

$$
\eta_{1} \equiv \frac{2 z-T\left(S_{2}+\sqrt{S_{2}^{2}-4 D}\right)}{2 a \alpha \varphi(\varepsilon-1)(1-N)} \text { and } \eta_{2} \equiv \frac{2 z-T\left(S_{2}-\sqrt{S_{2}^{2}-4 D}\right)}{2 a \alpha \varphi(\varepsilon-1)(1-N)}
$$

Proposition 23 (Hopf) Let $\varepsilon>1$ and $1-2 N<a-\rho<0$. A limit cycle (through a Hopf bifurcation) arises near $N_{2}$ at $\eta=\eta_{1}$.

We introduce now a critical value to have a codimension-two bifurcation:

$$
\eta_{B T} \equiv 2 \frac{1-\alpha}{\alpha} \frac{\rho+\delta}{\varepsilon-1}
$$

Proposition 24 (Bogdanov-Takens) Let $\varepsilon>1$. A Bogdanov-Takens bifurcation occurs if and only if $\eta=\eta_{B T}$ and $b=b^{*}$.

Proposition 25 (Gavrilov-Guckenheimer) A Gavrilov-Guckenheimer bifurcation is impossible in this example.

Proposition 26 (double-Hopf) In this example, a double-Hopf bifurcation is impossible.

To study the stability of the limit cycle around $N_{2}$, we perform a numerical simulation under the following calibration:

\begin{tabular}{||l||l||l||l||l||l||l||l||}
\hline \hline Parameter & $A$ & $\alpha$ & $\rho$ & $\delta$ & $a$ & $\gamma$ & $\varepsilon$ \\
\hline \hline Value & 1 & 0.3 & 0.2 & 0.1 & 0.1 & 0.1 & 2 \\
\hline \hline
\end{tabular}

As above, we simplify the computation setting $A, \alpha, \rho$ and $\delta$ such that $k^{*}=1$. We have fixed all the values as in the previous example except for $\gamma$ which is a new parameter. 
According to Proposition 23, $\varepsilon>1$ and $\rho>a$ are necessary (but not sufficient) conditions to get a Hopf bifurcation, while, according to Proposition 24 $\varepsilon>1$ is a necessary (but not sufficient) condition to obtain a Bogdanov-Takens bifurcation.

Calibration (23) entails $\left(b^{*}, \eta_{B T}\right)=(0.25,1.4)$.

We draw the bifurcation curves using the MATCONT package for MATLAB under calibration (23). In Fig. 2, $L P, H$ and $B T$ stand for Limit Point (elementary saddle-node), Hopf and Bogdanov-Takens. These points are computed and represented by MATCONT when an elementary saddle-node, a Hopf and a Bogdanov-Takens bifurcation occur near the steady state.

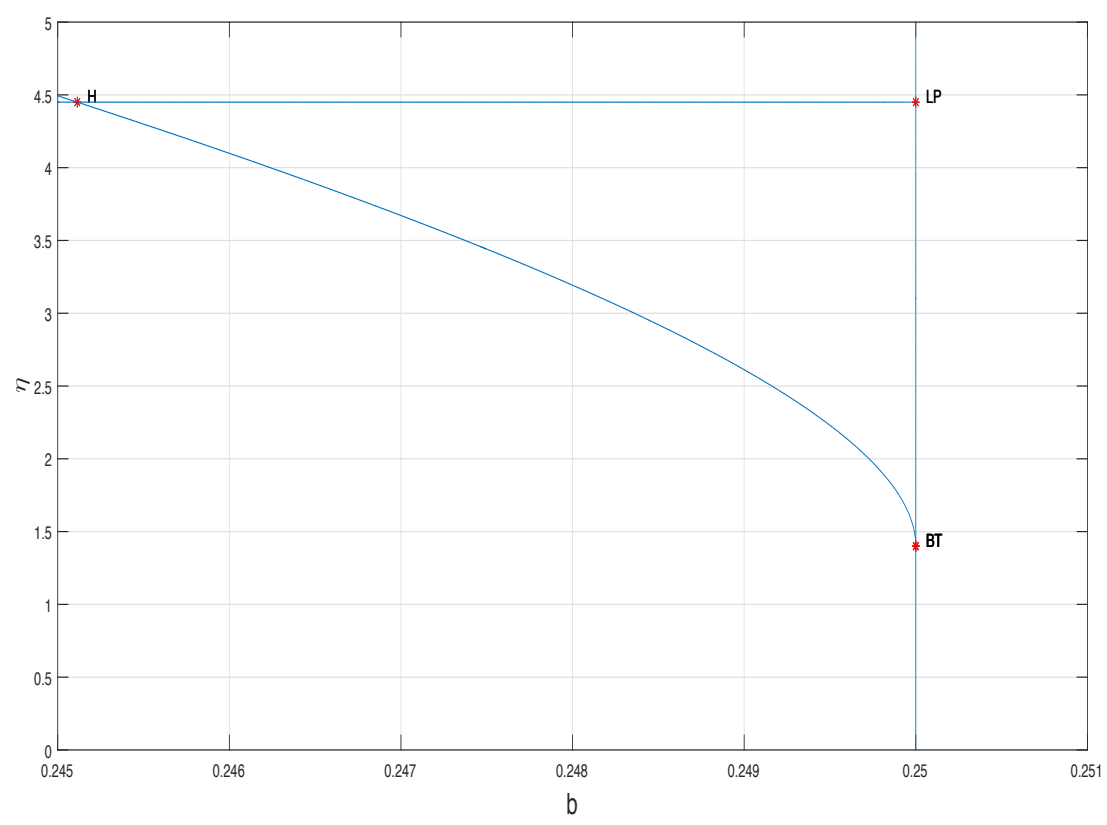

Fig. 2. Bifurcation curves in the $(b, \eta)$-plane.

Since a Hopf bifurcation occurs only around the higher steady state, the continuation exercise focuses only on $N_{2}$. The curve represents the locus of Hopf bifurcations: $\left\{\left(b, \eta_{1}(b)\right)\right\}$, where:

$$
\eta_{1}(b)=\frac{2 z(b)-T(b)\left[S_{2}+\sqrt{S_{2}^{2}(b)-4 D(b)}\right]}{2 a \alpha \varphi(\varepsilon-1)[1-N(b)]}
$$

We start by considering an arbitrary value $b_{0}=0.245<0.25=b^{*}$ (Proposition 22). We fix $\eta=4.45$ which is precisely the Hopf critical value when $b=0.24511$. 
For any $\eta$, the elementary saddle-node bifurcation value for $b$ is $b^{*}=0.25$ (the line $L P-B T$ is vertical because $b^{*}$ does not depend on $\eta$ ). In particular, the Limit Point (LP) corresponding to $\eta=4.45$ is $L P=(0.25,4.45)$.

Increasing $b$ from $b_{0}=0.245$ to $b^{*}=0.25$, we obtain all the Hopf bifurcation points $\left(b, \eta_{1}(b)\right)$ along the curve $C \equiv\left\{\left(b, \eta_{1}(b)\right)\right\}_{b \in\left[b_{0}, b^{*}\right]}$ from $H$ to $B T$. In the range $\left[b_{0}, b^{*}\right) \ni b$, we have two distinct steady states. When $b$ attains the maximal value $b^{*}$ these two steady states coalesce and the Hopf bifurcation point $\left(b, \eta_{1}(b)\right)$ reaches the ending point $B T$ along the curve $C$ while the economy experiences a Bogdanov-Takens bifurcation.

At the Hopf bifurcation point $(H)$, the steady state is given by: $(\mu, k, N, P)=$ $(15.073291,1,0.56990084,2.4511387)$ with eigenvalues: $\lambda_{1}=-0.391984, \lambda_{2}=$ $0.0978259 i=-\lambda_{3}$ and $\lambda_{4}=0.352183$.

The corresponding first Lyapunov coefficient is given by $l_{1}=1.283382 *$ $10^{-3}>0$. Its positivity means that the Hopf bifurcation is subcritical, that is the limit cycle arising near $N_{2}$ is unstable (Fig. 3).

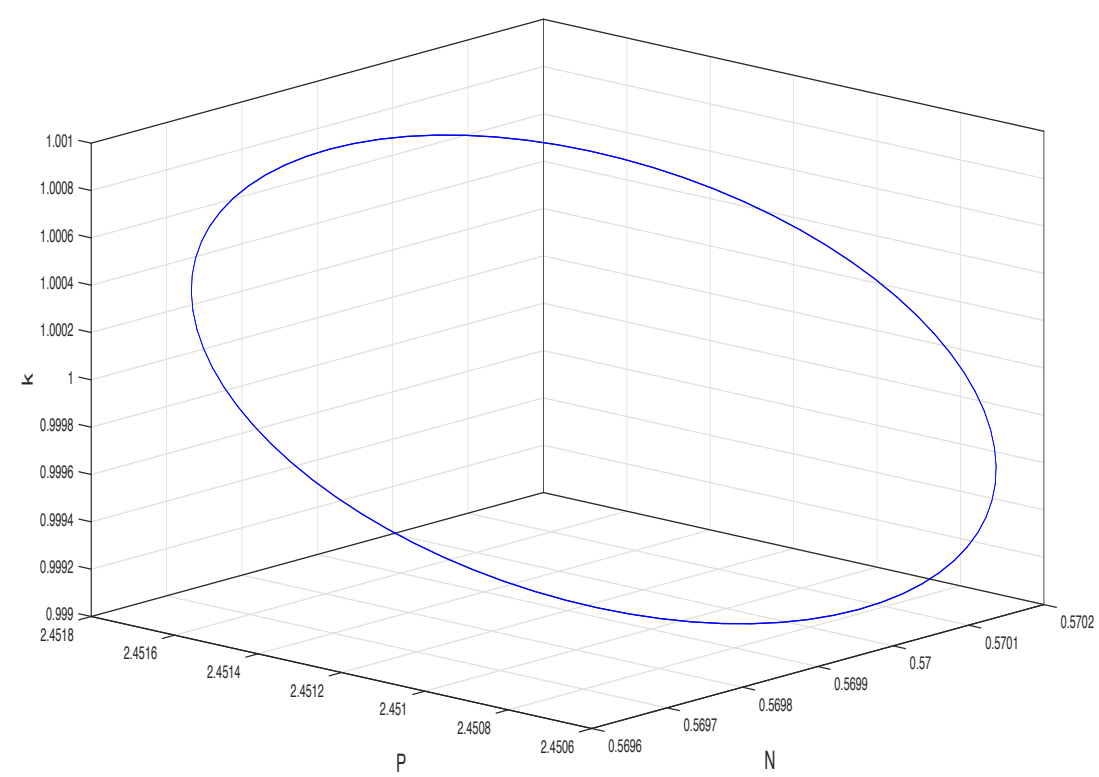

Fig. 3. Unstable limit cycle around $N_{2}$.

At the saddle-node bifurcation $(L P)$, the steady state becomes: $(\mu, k, N, P)=$ $(26.997759,1,0.5,2.5)$ with eigenvalues: $\lambda_{1}=-0.36618, \lambda_{2}=0, \lambda_{3}=0.233176+$ $0.0437607 i$ and $\lambda_{4}=0.233176-0.0437607 i$.

For now, we have considered codimension-one bifurcations (elementary saddlenode and Hopf). 
According to Proposition 24, we consider the codimension two. The BogdanovTakens $(B T)$ bifurcation arises at $(b, \eta)=\left(b^{*}, \eta_{B T}\right)=(0.25,1.4)$. The BogdanovTakens bifurcation occurs when conditions for the elementary saddle-node bifurcation and for the Hopf bifurcation meet each other.

At the Bogdanov-Takens point, the steady state becomes: $(\mu, k, N, P)=$ $(3.258,1,0.5,2.5)$ with real eigenvalues: $\lambda_{1}=-0.292053, \lambda_{2}=\lambda_{3}=0$ and $\lambda_{4}=0.392053$.

As in Kuznetsov et al. (2014), at the Bogdanov-Takens point, the orbit describes a parasitic loop near the saddle-point (Fig. 4). The parasitic loop typically arises when the limit cycle and the saddle-point coalesce.

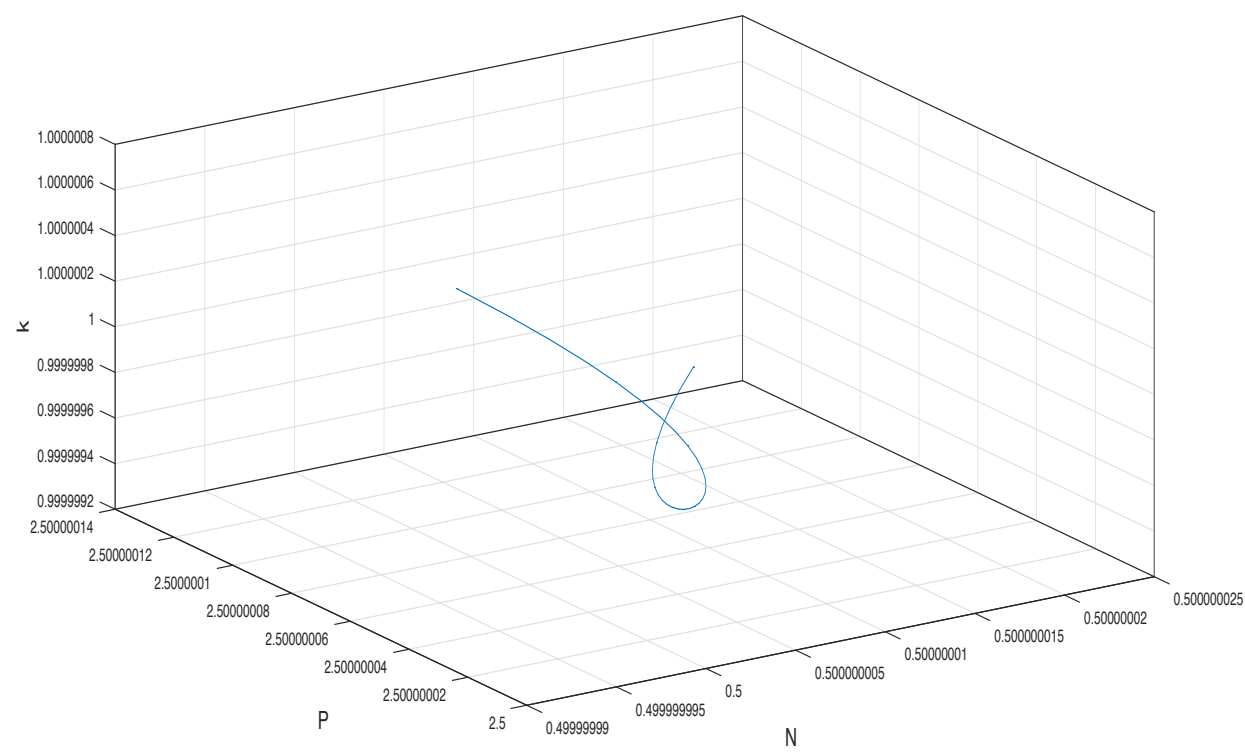

Fig. 4. Parasitic loop.

\section{Conclusion}

We have considered three and four-dimensional continuous-time dynamical systems and provided necessary and sufficient conditions for local bifurcations based on the sum of the minors of the Jacobian matrix. We have characterized not only the local bifurcations of codimension one but also those of codimension two. Our method is general and tractable. To illustrate the tractability of our approach, we have presented two extended Ramsey models of environmental economics: in the first one, pollution is an externality with a negative impact on household's utility and supercritical (stable) limit cycles arise; in the 
second one, the natural capital has a positive impact on utility and subcritical (unstable) limit cycles occur. In both the models, the analytical results are complemented with numerical simulations.

\section{Appendix}

\section{Proof of Proposition 1}

If a real eigenvalue crosses zero, we have $D=0$. Conversely, let $D=0$. If all the eigenvalues are real, then one of them is zero. If one is real, say $\lambda_{1}$, and two are nonreal, say $\lambda_{2}$ and $\lambda_{3}$, then $\lambda_{2}$ and $\lambda_{3}$ are conjugated with $\lambda_{2} \lambda_{3}>0$ (indeed $\lambda_{2} \lambda_{3}=a^{2}+b^{2}=0$ implies $a=b=0$ and, therefore, $\lambda_{2}=\lambda_{3}=0 \in \mathbb{R}$, a contradiction). Thus, $D=\lambda_{1} \lambda_{2} \lambda_{3}=0$ implies $\lambda_{1}=0$.

\section{Proof of Proposition 2}

Necessity In a three-dimensional dynamical system, we require at the bifurcation value: $\lambda_{1}=i b=-\lambda_{2}$ with no generic restriction on $\lambda_{3}$ (see Bosi and Ragot (2011) or Kuznetsov (1998) among others). The characteristic polynomial of $J$ is given by: $P(\lambda)=\left(\lambda-\lambda_{1}\right)\left(\lambda-\lambda_{2}\right)\left(\lambda-\lambda_{3}\right)=\lambda^{3}-T \lambda^{2}+S \lambda-D$. Using $\lambda_{1}=i b=-\lambda_{2}$, we find $D=b^{2} \lambda_{3}, S=b^{2}, T=\lambda_{3}$. Thus, $D=S T$ and $S>0$.

Sufficiency In the case of a three-dimensional system, one eigenvalue is always real, the others two are either real or nonreal and conjugated. Let us show that, if $D=S T$ and $S>0$, these eigenvalues are nonreal with zero real part and, hence, a Hopf bifurcation generically occurs.

We observe that $D=S T$ implies

$$
\lambda_{1} \lambda_{2} \lambda_{3}=\left(\lambda_{1} \lambda_{2}+\lambda_{1} \lambda_{3}+\lambda_{2} \lambda_{3}\right)\left(\lambda_{1}+\lambda_{2}+\lambda_{3}\right)
$$

or, equivalently,

$$
\left(\lambda_{1}+\lambda_{2}\right)\left[\lambda_{3}^{2}+\left(\lambda_{1}+\lambda_{2}\right) \lambda_{3}+\lambda_{1} \lambda_{2}\right]=0
$$

This equation holds if and only if $\lambda_{1}+\lambda_{2}=0$ or $\lambda_{3}^{2}+\left(\lambda_{1}+\lambda_{2}\right) \lambda_{3}+\lambda_{1} \lambda_{2}=0$. Solving this second-degree equation for $\lambda_{3}$, we find $\lambda_{3}=-\lambda_{1}$ or $-\lambda_{2}$. Thus, (24) holds if and only if $\lambda_{1}+\lambda_{2}=0$ or $\lambda_{1}+\lambda_{3}=0$ or $\lambda_{2}+\lambda_{3}=0$. Without loss of generality, let $\lambda_{1}+\lambda_{2}=0$ with, generically, $\lambda_{3} \neq 0$, a real eigenvalue. Since $S>0$, we have also $\lambda_{1}=-\lambda_{2} \neq 0$. We obtain $T=\lambda_{3} \neq 0$ and $S=D / T=\lambda_{1}$ $\lambda_{2}=-\lambda_{1}^{2}>0$. This is possible only if $\lambda_{1}$ is nonreal. If $\lambda_{1}$ is nonreal, $\lambda_{2}$ is conjugated, and, since $\lambda_{1}=-\lambda_{2}$, they have a zero real part.

\section{Proof of Proposition 4}

A Bogdanov-Takens bifurcation arises if and only if two real eigenvalues cross zero (say, $\lambda_{1}=\lambda_{2}=0$ ). Therefore, $D=\lambda_{1} \lambda_{2} \lambda_{3}=0$ and $S=\lambda_{1} \lambda_{2}+$ $\lambda_{1} \lambda_{3}+\lambda_{2} \lambda_{3}=0$. Conversely, if $D=0$, at least one eigenvalue is zero, say $\lambda_{1}$. $S=\lambda_{2} \lambda_{3}=0$ implies that another eigenvalue is zero, say $\lambda_{2}$.

\section{Proof of Proposition 6}

Necessity $T=0+b i-b i=0$ and $D=0 * b^{2}=0$. Moreover, $S=b^{2}>0$. Definition 5 applies. 
Sufficiency Conversely, if $D=0$, there exists, at least, one eigenvalue equal to zero $\left(\lambda_{1}=0\right)$. Since $\lambda_{1}=0$ and $T=0$, then $\lambda_{2}=-\lambda_{3}$. If $\lambda_{2}$ and $\lambda_{3}$ are real, $\lambda_{2}=-\lambda_{3}$ implies $S=\lambda_{2} \lambda_{3} \leq 0$, a contradiction. Then, $\lambda_{2}$ and $\lambda_{3}$ are nonreal and conjugated. Since $\lambda_{2}=-\lambda_{3}$, they have a zero real part: $\lambda_{2}=b i=-\lambda_{3}$. Notice that, in this case, $S=\lambda_{2} \lambda_{3}=b^{2}>0$.

Proof of Proposition 7

Profit maximization $\max _{K_{j}, L_{j}}\left(A K_{j}^{\alpha} L_{j}^{1-\alpha}-r K_{j}-w L_{j}\right)$ implies the following first-order conditions:

$$
r=\alpha A k^{\alpha-1} \text { and } w=(1-\alpha) A k^{\alpha}
$$

where $k=K_{j} / L_{j}$. The average productivity is given by $y \equiv Y_{j} / L_{j}=A k^{\alpha}$.

To solve the utility maximization problem, we build the constant-value Hamiltonian $H \equiv\left(c P^{-\eta}\right)^{1-\varepsilon} /(1-\varepsilon)+\mu[(r-\delta) k+w-c]$, where $\mu$ denotes the multiplier. The first-order conditions are given by a static arbitrage: $c=$ $\mu^{-1 / \varepsilon} P^{\eta(1-1 / \varepsilon)}$, and two dynamic equations: $\dot{\mu}=\mu(\rho+\delta-r)$ and $\dot{h}=(r-\delta) h+$ $w-c$, jointly with the usual transversality condition.

Noticing that $h=k$ and replacing the first-order conditions for profit maximization in the first-order conditions for utility maximization, we obtain the general equilibrium system (4)-(6).

Proof of Proposition 8

The steady state $(\mu, k, P)^{*}$ is solution to system $\dot{\mu}=\dot{k}=\dot{P}=0 . \dot{\mu}=0$ has a unique solution $k^{*}$. Replacing this solution in $\dot{k}=\dot{P}=0$, we find a unique steady state.

\section{Proof of Lemma 9}

In order to capture the dynamics near the steady state, we linearize the system (4)-(6) around $(\mu, k, P)^{*}$. The Jacobian matrix is given by

$$
J \equiv\left[\begin{array}{lll}
\frac{\partial f_{1}}{\partial \mu} & \frac{\partial f_{1}}{\partial k} & \frac{\partial f_{1}}{\partial P} \\
\frac{\partial f_{2}}{\partial \mu} & \frac{\partial f_{2}}{\partial k} & \frac{\partial f_{2}}{\partial P} \\
\frac{\partial f_{3}}{\partial \mu} & \frac{\partial f_{3}}{\partial k} & \frac{\partial f_{3}}{\partial P}
\end{array}\right]=\left[\begin{array}{ccc}
0 & (1-\alpha)(\rho+\delta) \frac{\mu^{*}}{k^{*}} & 0 \\
\varphi \frac{k^{*}}{\mu^{*}} & \rho & -\varphi \eta(\varepsilon-1) \frac{a}{b} \\
0 & b & -a
\end{array}\right]
$$

We compute the sums of minors of order one (trace $T$ ), two $(S)$ and three (determinant $D$ ) using (2).

\section{Proof of Proposition 10}

$\alpha \in(0,1)$ implies $D>0$. Propositions 1,4 and 6 apply.

\section{Proof of Proposition 12}

Consider Proposition 2. $D=S T$ if and only if $\eta=\eta_{H}$. In addition, if $\rho>a$, $T>0$. Since $D>0$, then $S>0$ at $\eta=\eta_{H}$. Finally, $\varepsilon>1$ and $\rho>a$ imply $\eta_{H}>0$, an economically meaningful value.

\section{Proof of Proposition 13}

If a real eigenvalue crosses zero, we have $D=0$. Conversely, let $D=0$. If all the eigenvalues are real, then one of them is zero. If two are real, say $\lambda_{1}$ and $\lambda_{2}$, and two are nonreal, say $\lambda_{3}$ and $\lambda_{4}$, then $\lambda_{3}$ and $\lambda_{4}$ are conjugated with, generically, $\lambda_{3} \lambda_{4}>0$. Thus, $\lambda_{1} \lambda_{2}=0$, that is one eigenvalue is zero. When all the eigenvalues are nonreal, generically $\lambda_{1} \lambda_{2} \lambda_{3} \lambda_{4}>0$ : this case is incompatible with $D=0$. 


\section{Proof of Proposition 14}

A Hopf bifurcation generically arises if and only if $\lambda_{1}=i b$ and $\lambda_{2}=-i b$ and $b \neq 0$ or, equivalently, $\lambda_{1}+\lambda_{2}=0$ and $\lambda_{1} \lambda_{2}=p>0$ for some pair of eigenvalues $\lambda_{1}$ and $\lambda_{2}$, and some $p \equiv b^{2}>0$.

If $\lambda_{h}+\lambda_{i}=0$ and $\lambda_{h} \lambda_{i}>0$, according to (9)-(12), a Hopf bifurcation implies $T=\lambda_{j}+\lambda_{k}, S_{2}=b^{2}+\lambda_{j} \lambda_{k}, S_{3}=b^{2} T, D=b^{2} \lambda_{j} \lambda_{k}$, where $(h, i, j, k)$ is a permutation of $(1,2,3,4)$, and, therefore,

$$
\begin{aligned}
& S_{2}=p+\frac{D}{p} \\
& S_{3}=p T
\end{aligned}
$$

hold for some $p>0$. Remark that (26) are (27) are only necessary conditions for a Hopf bifurcation to occur.

The question we raise is whether (26) and (27) with $p>0$ are also (generic) sufficient conditions for a Hopf bifurcation to arise.

A Hopf bifurcation generically arises if and only if $\lambda_{1}+\lambda_{2}=0$ and $\lambda_{1} \lambda_{2}=p$ for some pair of eigenvalues and some $p>0$.

Assume that (28) and (29) hold but, for any pair of eigenvalues $\lambda_{1}$ and $\lambda_{2}$, we have $\lambda_{1}+\lambda_{2} \neq 0$ or $\lambda_{1} \lambda_{2} \leq 0$.

Equations (26) and (27) are equivalent to

$$
\begin{aligned}
B+D+A C & =p+\frac{B D}{p} \\
A D+B C & =p(A+C)
\end{aligned}
$$

with $p>0$ and $A \equiv \lambda_{h}+\lambda_{i}, B \equiv \lambda_{h} \lambda_{i}, C \equiv \lambda_{j}+\lambda_{k}$ and $D \equiv \lambda_{j} \lambda_{k}$.

We consider two cases.

(1) If $A=0$, then $(29)$ implies $(B-p) C=0$, that is $B=p>0$, a contradiction (indeed, for any pair of eigenvalues, if their sum is zero, their product is non-positive).

(2) If $A \neq 0$, then (29) implies

$$
D=p+\frac{p-B}{A} C
$$

Replacing $D$ in (28), we get

$$
A^{2} C=-\frac{(p-B)^{2}}{p} C
$$

If $C=0$, then $D>0$, a contradiction (apply the same argument of point (1) with $C$ and $D$ instead of $A$ and $B$ respectively). Thus, $0<A^{2}=-(p-B)^{2} / p \leq 0$, a contradiction.

Thus, (28) and (29) imply, for some pair of eigenvalues, $\lambda_{1}+\lambda_{2}=0$ and $\lambda_{1} \lambda_{2}=p>0$, that is, generically, a Hopf bifurcation.

Summing up, a Hopf bifurcation generically arises if and only if (26) are (27) hold for some $p>0$. 
Finally, notice that $p$ is an arbitrary positive number in order to obtain (13).

\section{Proof of Proposition 15}

Necessity According to (11) and (12), $\lambda_{1}=\lambda_{2}=0$ implies $S_{3}=D=0$.

Sufficiency $D=0$ implies, without loss of generality, $\lambda_{1}=0$. According to (11), we obtain $S_{3}=\lambda_{2} \lambda_{3} \lambda_{4}=0$ and, thus, without loss of generality, $\lambda_{2}=0$.

\section{Proof of Proposition 16}

Necessity If $\lambda_{1}=0, \lambda_{3}=i d$ and $\lambda_{4}=-i d$, according to (9)-(12), we obtain $T=\lambda_{2}, S_{2}=d^{2}, S_{3}=d^{2} \lambda_{2}$ and $D=0$. These equations imply $D=0$ and $S_{3}=T S_{2}$ with $S_{2}>0$.

Sufficiency If $D=0$, then $\lambda_{1}=0$ without loss of generality. According to (9)-(12), this entails $T=\lambda_{2}+\lambda_{3}+\lambda_{4}, S_{2}=\lambda_{2} \lambda_{3}+\lambda_{2} \lambda_{4}+\lambda_{3} \lambda_{4}$ and $S_{3}=\lambda_{2} \lambda_{3} \lambda_{4}$. Hence, $S_{3}=T S_{2}$ becomes $\lambda_{2} \lambda_{3} \lambda_{4}=\left(\lambda_{2}+\lambda_{3}+\lambda_{4}\right)\left(\lambda_{2} \lambda_{3}+\lambda_{2} \lambda_{4}+\lambda_{3} \lambda_{4}\right)$ or, equivalently, $\left(\lambda_{2}+\lambda_{3}\right)\left(\lambda_{2}+\lambda_{4}\right)\left(\lambda_{3}+\lambda_{4}\right)=0$. Let, without loss of generality, $\lambda_{3}+\lambda_{4}=0$. Then, $\lambda_{3}=-\lambda_{4}$ and $S_{2}=\lambda_{3} \lambda_{4}$. Since $S_{2}>0$, we obtain $\lambda_{3}=i d$ and $\lambda_{4}=-i d$ for some real number $d$.

\section{Proof of Proposition 18}

Necessity We observe that $\lambda_{1}+\lambda_{2}=\lambda_{3}+\lambda_{4}=0$ and that $\lambda_{1} \lambda_{2}=b^{2}$ and $\lambda_{3} \lambda_{4}=d^{2}$. According to (9)-(12), we obtain $T=0, S_{2}=b^{2}+d^{2}>0, S_{3}=0$ and $D=b^{2} d^{2}>0$. Moreover, $S_{2}^{2}-4 D=\left(b^{2}+d^{2}\right)^{2}-4 b^{2} d^{2}=\left(b^{2}-d^{2}\right)^{2} \geq 0$.

Sufficiency $T=0$ implies $\lambda_{3}+\lambda_{4}=-\left(\lambda_{1}+\lambda_{2}\right)$. This with $S_{3}=0$ imply

$$
S_{3}=\lambda_{1} \lambda_{2}\left(\lambda_{3}+\lambda_{4}\right)+\left(\lambda_{1}+\lambda_{2}\right) \lambda_{3} \lambda_{4}=\left(\lambda_{1}+\lambda_{2}\right)\left(\lambda_{3} \lambda_{4}-\lambda_{1} \lambda_{2}\right)=0
$$

Then, (1) $\lambda_{1}+\lambda_{2}=0$ (and, so, $\lambda_{3}+\lambda_{4}=0$ ) or (2) $\lambda_{1} \lambda_{2}=\lambda_{3} \lambda_{4}$.

Let us to prove a preliminary result. The case of the following four eigenvalues:

$$
a+b i, a-b i,-a+b i,-a-b i
$$

with $a, b \neq 0$ is excluded. Indeed,

$$
\begin{aligned}
S_{2}^{2}-4 D & =\left(\lambda_{1} \lambda_{2}+\lambda_{1} \lambda_{3}+\lambda_{1} \lambda_{4}+\lambda_{2} \lambda_{3}+\lambda_{2} \lambda_{4}+\lambda_{3} \lambda_{4}\right)^{2}-4 \lambda_{1} \lambda_{2} \lambda_{3} \lambda_{4} \\
& =-16 a^{2} b^{2}<0
\end{aligned}
$$

(notice that the order of the eigenvalues does not matter: whatever permutation $(1,2,3,4) \rightarrow(h, i, j, k)$ gives the same result $)$.

(1) If $\lambda_{1}+\lambda_{2}=\lambda_{3}+\lambda_{4}=0$, then $S_{2}=\lambda_{1} \lambda_{2}+\lambda_{3} \lambda_{4}=-\left(\lambda_{1}^{2}+\lambda_{3}^{2}\right)$. Thus, $S_{2}>0$ implies $\lambda_{1}^{2}+\lambda_{3}^{2}<0$. Then, one of the two eigenvalues is nonreal, say $\lambda_{1}$.

If $\lambda_{3}^{2}$ is real, then, $\lambda_{1}^{2}$ is real as well and, therefore, $\lambda_{1}=b i$ for some real number $b \neq 0$. Then, $\lambda_{2}=-b i$ and $D=\lambda_{1} \lambda_{2} \lambda_{3} \lambda_{4}=b^{2} \lambda_{3} \lambda_{4}>0$ implies $\lambda_{3} \lambda_{4}>0$. This, jointly with $\lambda_{3}=-\lambda_{4}$ entails $\lambda_{3}=d i$ and $\lambda_{4}=-d i$ for some real number $d \neq 0$.

If $\lambda_{3}^{2}$ is nonreal, then $\lambda_{3}$ is nonreal. Then, $\lambda_{1}, \lambda_{2}, \lambda_{3}$ and $\lambda_{4}$ are all nonreal and pairwise conjugated. If $\lambda_{1}$ is conjugated with $\lambda_{2}$, we have $\lambda_{1}=-\lambda_{2}=b i$ for some real number $b \neq 0$. Since $\lambda_{3}$ and $\lambda_{4}$ are also conjugated, we have $\lambda_{3}=-\lambda_{4}=d i$ for some real number $d \neq 0$. If $\lambda_{1}$ is conjugated with $\lambda_{3}$, then 
$\lambda_{2}$ is conjugated with $\lambda_{4}$. In this case, let $\lambda_{1}=a+b i, \lambda_{3}=a-b i, \lambda_{2}=c-d i$, $\lambda_{4}=c+d i$ for some real numbers $b, d \neq 0$. Since $\lambda_{1}-\lambda_{3}=\lambda_{4}-\lambda_{2}$, we obtain $b=d$. Moreover, $\lambda_{1}+\lambda_{2}=0$ implies $a+b i+c-b i=0$, that is $c=-a$. Thus, $\lambda_{1}=a+b i, \lambda_{2}=-a-b i, \lambda_{3}=a-b i, \lambda_{4}=-a+b i$. If $a \neq 0$, then $S_{2}^{2}<4 D$ because of (31), a contradiction. Then, $\lambda_{1}=\lambda_{4}=b i$ and $\lambda_{2}=\lambda_{3}=-b i$, that is a double-Hopf bifurcation.

(2) If $\lambda_{1} \lambda_{2}=\lambda_{3} \lambda_{4}$, then

$$
S_{2}=\lambda_{1} \lambda_{2}+\lambda_{3} \lambda_{4}+\left(\lambda_{1}+\lambda_{2}\right)\left(\lambda_{3}+\lambda_{4}\right)=2 \lambda_{1} \lambda_{2}-\left(\lambda_{1}+\lambda_{2}\right)^{2}=-\lambda_{1}^{2}-\lambda_{2}^{2}>0
$$

Then, $\lambda_{1}$ or $\lambda_{2}$ is nonreal.

Similarly, we get $S_{2}=-\lambda_{3}^{2}-\lambda_{4}^{2}>0$. Then, $\lambda_{3}$ or $\lambda_{4}$ is nonreal.

Without loss of generality let $\lambda_{1}=a+b i$ and $\lambda_{3}=c+d i$ be nonreal $(b, d \neq 0)$.

(2.1) If $\lambda_{1}$ and $\lambda_{3}$ are not conjugated, then all the eigenvalues are nonreal: $\lambda_{1}=a+b i, \lambda_{i}=a-b i, \lambda_{3}=c+d i, \lambda_{j}=c-d i . T=0$ implies $c=-a$. Using $\lambda_{1}=a+b i, \lambda_{i}=a-b i, \lambda_{3}=-a+d i, \lambda_{j}=-a-d i$ with $i \neq j$, and $\lambda_{1} \lambda_{2}=\lambda_{3}$ $\lambda_{4}$, we obtain, if $a \neq 0, d= \pm b$ and, therefore, the four eigenvalues (30). Since $a \neq 0$, then $S_{2}^{2}<4 D$ because of (31), a contradiction. Then $a=0$ and, thus, we obtain $\lambda_{1}=b i, \lambda_{i}=-b i, \lambda_{3}=d i, \lambda_{j}=-d i$, that is a double-Hopf bifurcation.

(2.2) If $\lambda_{1}$ and $\lambda_{3}$ are conjugated with $a \neq 0, \lambda_{1} \lambda_{2}=\lambda_{3} \lambda_{4}$ implies that $\lambda_{2}$ or $\lambda_{4}$ is nonreal. In this case, the other eigenvalue is conjugated and nonreal. Thus, we have $\lambda_{1}=a+b i, \lambda_{2}=x+y i, \lambda_{3}=a-b i, \lambda_{4}=x-y i$. $T=0$ implies $x=-a$. Thus, $\lambda_{1}=a+b i, \lambda_{2}=-a+y i, \lambda_{3}=a-b i, \lambda_{4}=-a-y i . \lambda_{1} \lambda_{2}=\lambda_{3}$ $\lambda_{4}$ implies $b=y$. Then, $\lambda_{1}=a+b i, \lambda_{2}=-a+b i, \lambda_{3}=a-b i, \lambda_{4}=-a-b i$. Since $a \neq 0$, then $S_{2}^{2}<4 D$ because of (31), a contradiction.

If $\lambda_{1}$ and $\lambda_{3}$ are conjugated with $a=0$, we have $\lambda_{1}=-\lambda_{3}=b i . \quad T=0$ and $D>0$ imply $\lambda_{2}=-\lambda_{4}$ and $\lambda_{2} \lambda_{4}>0$, that is $\lambda_{2}=-\lambda_{4}=d i$ for some real number $d \neq 0$. According to Definition 17, we have a double-Hopf bifurcation.

\section{Proof of Proposition 19}

The first-order conditions of profit maximization are still given by (25).

To solve the utility maximization problem, we build the constant-value Hamiltonian $H \equiv\left(c N^{\eta}\right)^{1-\varepsilon} /(1-\varepsilon)+\mu[(r-\delta) h+w-c]$, where $\mu$ denotes the multiplier. The first-order conditions are given by a static arbitrage: $c=$ $\mu^{-1 / \varepsilon} N^{\eta(1 / \varepsilon-1)}$, and two dynamic equations: $\dot{\mu}=\mu(\rho+\delta-r)$ and $\dot{h}=(r-\delta) h+$ $w-c$, jointly with the usual transversality condition.

Noticing that $h=k$ and replacing the first-order conditions for profit maximization in the first-order conditions for utility maximization, we obtain the general equilibrium system (4)-(6).

\section{Proof of Proposition 20}

The steady states are given by $\dot{\mu}=\dot{k}=\dot{N}=\dot{P}=0$. Equations (14) and (17) imply (19) and (20) respectively. (16) entails (21) and (22). Replacing $k^{*}$ in equation (15) gives (18). Finally, $1-4 \gamma P^{*}>0$ if and only if $b<b^{*}$.

Proof of Lemma 21

We linearize the system (14)-(17) around one of the two steady states, say 
$N^{*}$. The Jacobian matrix $J$ is given by

$$
\begin{aligned}
J & \equiv\left[\begin{array}{llll}
\frac{\partial f_{1}}{\partial \mu} & \frac{\partial f_{1}}{\partial k} & \frac{\partial f_{1}}{\partial N} & \frac{\partial f_{1}}{\partial P} \\
\frac{\partial f_{2}}{\partial \mu} & \frac{\partial f_{2}}{\partial k} & \frac{\partial f_{2}}{\partial N} & \frac{\partial f_{2}}{\partial P} \\
\frac{\partial f_{3}}{\partial \mu} & \frac{\partial f_{3}}{\partial k} & \frac{\partial f_{3}}{\partial N} & \frac{\partial f_{3}}{\partial P} \\
\frac{\partial f_{4}}{\partial \mu} & \frac{\partial f_{4}}{\partial k} & \frac{\partial f_{4}}{\partial N} & \frac{\partial f_{4}}{\partial P}
\end{array}\right] \\
& =\left[\begin{array}{cccc}
0 & (1-\alpha)(\rho+\delta) \frac{\mu}{k} & 0 & 0 \\
\varphi \frac{k}{\mu} & \rho & \alpha \varphi(1-N) \frac{a \eta}{b \gamma} \frac{\varepsilon-1}{\rho+\delta} & 0 \\
0 & 0 & 1-2 N & -\gamma \\
0 & b(\rho+\delta) & 0 & -a
\end{array}\right]
\end{aligned}
$$

We compute the sums of minors of order one (trace $T)$, two $\left(S_{2}\right)$, three $\left(S_{3}\right)$ and four (determinant $D$ ) using (8).

Proof of Proposition 22

According to Proposition 20, $N_{1}=N_{2}=1 / 2$ if and only if $b=b^{*}$ or, equivalently, $D=0$. We apply Proposition 13 . The saddle-node bifurcation is elementary because the two steady states disappear.

Proof of Proposition 23

Consider Proposition 14. $S_{2}=S_{3} / T+D T / S_{3}$ if and only if $\eta=\eta_{1}$ or $\eta=\eta_{2}$. In addition,

$$
\frac{S_{3}\left(\eta_{1}\right)}{T}=\frac{1}{2}\left(S_{2}+\sqrt{S_{2}^{2}-4 D}\right) \text { and } \frac{S_{3}\left(\eta_{2}\right)}{T}=\frac{1}{2}\left(S_{2}-\sqrt{S_{2}^{2}-4 D}\right)
$$

$1-2 N<a-\rho<0$ implies $T<0$ and $S_{2}<0$, that is $S_{3}\left(\eta_{2}\right) / T<0: T$ and $S_{3}\left(\eta_{2}\right)$ have opposite signs and, according to Proposition 14, limit cycles at $\eta=\eta_{2}$ are impossible. Conversely, $S_{3}\left(\eta_{1}\right) / T>0: T$ and $S_{3}\left(\eta_{2}\right)$ have the same sign and conditions of Proposition 14 are satisfied. Moreover, $\varepsilon>1$ and $1-2 N<a-\rho<0$ entail $\eta_{1}>0$. Finally, $1-2 N<0$ implies that a limit cycle occurs around $N_{2}$ but not around $N_{1}$.

Proof of Proposition 24

According to Proposition $15, D=0$ if and only if $b=b^{*}$ (that is $N_{1}=N_{2}=$ $1 / 2$ ). If $N=1 / 2$, then $S_{3}=0$ if and only if $\eta=\eta_{B T}$. Finally, $\varepsilon>1$ ensures that $\eta_{B T}>0$.

\section{Proof of Proposition 25}

According to Proposition 16, a Gavrilov-Guckenheimer bifurcation generically arises if and only if $D=0, S_{3}=T S_{2}$ and $S_{2}>0 . D=0$ if and only if $N_{1}=N_{2}=1 / 2$. But $N=1 / 2$ implies $S_{2}=-a \rho-\varphi(1-\alpha)(\rho+\delta)<0$. Then, the Gavrilov-Guckenheimer bifurcation is impossible.

\section{Proof of Proposition 26}

$T=0$ if and only if $1-2 N=a-\rho$, while $D>0$ if and only if $1-2 N>0$ implying that $a>\rho$ and, then, $S_{2}<0$. Proposition 18 applies. 


\section{References}

[1] Ayong Le Kama A. (2001). Sustainable growth, renewable resources and pollution. Journal of Economic Dynamics \& Control 25, 1911-1918.

[2] Barinci J.-P. and J.-P. Drugeon (2017). Assessing the local stability properties of discrete three-dimensional dynamical system: a geometrical approach with triangles and planes and an application with some cones. In: Nishimura K., A. Venditti and C.N. Yannelis eds., Sunspots and Non-Linear Dynamics: Essays in Honor of Jean-Michel Grandmont, Studies in Economic Theory 31, 15-39, Springer.

[3] Bosi S. and D. Desmarchelier (2016a). Limit cycles under a negative effect of pollution on consumption demand: the role of an environmental Kuznets curve. Forthcoming in Environmental and Resource Economics.

[4] Bosi S. and D. Desmarchelier (2016b). Natural cycles and pollution. Working Paper BETA 2016-53.

[5] Bosi S. and D. Desmarchelier (2017). A simple method to study local bifurcations of three and four-dimensional systems: characterizations and economic applications. Working Paper BETA 2017-07, Working Paper EPEE $17-01$.

[6] Bosi S. and L. Ragot (2011). Introduction to discrete-time dynamics. CLUEB, Bologna.

[7] Dockner E. and G. Feichtinger (1991). On the optimality of limit cycles in dynamic economic systems. Journal of Economics 53, 31-50.

[8] Grandmont J.-M. (2008). Nonlinear difference equations, bifurcations and chaos: an introduction. Research in Economics 62, 122-177.

[9] Heal G. (1982). The use of common property resources. In Explorations in Natural Resource Economics, The Johns Hopkins University Press for Resources for the Future, Baltimore.

[10] Kuznetsov A.P and J.V. Sedova (2012). Bifurcations of three- and fourdimensional maps: universal properties. $\quad$ 20, 26-43.

[11] Kuznetsov Y. (1998). Elements of Applied Bifurcation Theory. Springer, Applied Mathematical Sciences 112.

[12] Kuznetsov Y. A., H. G. E. Meijer, B. Al-Hdaibat and W. Govaerts (2014). Improved homoclinic predictor for Bogdanov-Takens bifurcation. International Journal of Bifurcation and Chaos 24, 1-14.

[13] Ramsey, F. (1928). A Mathematical Theory of Saving. Economic Journal 38, 543-559. 
[14] Wirl F. (2004). Sustainable growth, renewable resources and pollution: thresholds and cycles. Journal of Economic Dynamics $\& \mathcal{G}$ Control 28, 11491157. 